¿Existe una asociación entre la frecuencia del síndrome de Down y la de malformaciones congénitas del tubo neural en familias?

Recientemente se publicó que tanto las malformaciones congénitas del tubo neural (MCTN) como el síndrome de Down estaban asociados con determinadas alteraciones de la vía metabólica del folato y que, por lo tanto, podría esperarse que al menos algunos casos de una y otra afección tuvieran una causa común. Se informó, además, de que entre las familias israelíes en mayor riesgo de tener progenie con MCTN había más casos de síndrome de Down de lo esperado y que, por su parte, entre las familias ucranianas en mayor riesgo de tener descendencia con síndrome de Down se encontró un número de niños con MCTN mayor que en la población en general. (Barkai G, Arbuzova S, Berkenstadt M, Heifetz S, Cuckle H. Frequency of Down syndrome and neural-tube defects in the same family. Lancet 2003;361:1331-1335.)

El objetivo de esta investigación fue comprobar si la relación entre la incidencia de síndrome de Down y la de MCTN en una misma familia podía confirmarse en un estudio mayor que abarcara varios países de América del Sur.

Se estudió a 2421 casos de MCTN, 952 de hidrocefalia y 3095 de síndrome de Down de un total de 1583838 nacimientos registrados entre 1982 y 2000 como parte del Estudio Colaborativo Latinoamericano de Malformaciones Congénitas. En este estudio participaron hospitales de Argentina, Bolivia, Brasil, Chile, Colombia, Ecuador, Paraguay, Perú, Uruguay y Venezuela.

Se contó el número de casos de síndrome de Down que fuesen hermanos de los casos índice de MCTN o de hidrocefalia congénita, y se estimó el número esperado de ellos según la edad de la madre. También se registró el número de casos de MCTN o hidrocefalia congénita entre los hermanos de los casos índice de síndrome de Down y se calculó el número esperado de acuerdo con la prevalencia de esas afecciones en la población estudiada.

Se calculó la frecuencia esperada de niños con síndrome de Down para cada embarazo anterior al nacimiento de un niño con MCTN o hidrocefalia de la misma madre. El número esperado de casos con MCTN o hidrocefalia para todos los embarazos anteriores al nacimiento de un niño con síndrome de Down de una misma madre se calculó a partir de la prevalencia combinada de nacimientos con MCTN y con hidrocefalia en la población estudiada $(0,00213)$.

Se encontró a 5 niños (de 5,13 esperados) con síndrome de Down entre los 5404 hermanos o medio hermanos de los 3373 casos índice de MCTN o de hidrocefalia congénita. Asimismo, se detectó a 12 recién nacidos (de 17,18 esperados) con MCTN entre los 8066 hermanos o medio hermanos de los 3095 casos índice de síndrome de Down. No se encontraron diferencias significativas entre el número de observaciones y el número de casos esperados.

El riesgo de MCTN observado entre los medio hermanos maternos de los casos índice de ese tipo de malformación fue la mitad del observado entre los hermanos que eran hijos de los mismos padres, tal como se esperaba, debido a la disminución de la consanguinidad. Esta relación también se observó entre los hermanos y medio hermanos de los casos índice de hidrocefalia congénita. La recurrencia cruzada (MCTN entre los hermanos de los niños con hidrocefalia) demostró que el riesgo entre los hermanos y medio hermanos maternos era el mismo y no difería del riesgo observado en la población en general.

En la población sudamericana estudiada tampoco se confirmó que en una misma familia hubiese una frecuencia de síndrome de Down y de MCTN mayor de la esperada.

El origen común atribuido al síndrome de Down y a las MCTN podría generar importantes medidas de prevención, por lo que la posible asociación entre los factores de riesgo de ambas afecciones debe ser objeto de estudio en otras poblaciones y en condiciones diferentes. (Amorim MR, Castilla EE, Orioli IM. Is there a familial link between Down's syndrome and neural tube defects? Population and familial survey. BMJ 2004;328:84-87.)

\section{Los diabéticos deben controlar mejor ciertos parámetros clínicos para evitar complicaciones vasculares}

La diabetes mellitus es un factor de riesgo de trastornos vasculares que pueden conducir a retinopatías, neuropatías, nefropatías, enfermedad coronaria y amputaciones de las extremidades inferiores. Numerosos ensayos clínicos han demostrado que es posible prevenir estas complicaciones si el paciente diabético mantiene su tensión arterial, 
glucemia y colesterol sérico total por debajo de ciertos valores máximos recomendados (tensión sistólica, $130 \mathrm{mmHg}$, tensión diastólica, $80 \mathrm{mmHg}$; glucohemoglobina $\left(\mathrm{HbA}_{1 \mathrm{c}}\right), 7 \%$; y colesterol sérico total, $200 \mathrm{mg} / \mathrm{dL}$ ). Se desconoce, sin embargo, qué porcentaje de los pacientes con diabetes diagnosticada logran un control clínico adecuado y por tal motivo un grupo de investigadores del Centro Nacional de Estadísticas de Salud en Hyattsville, Maryland, Estados Unidos, realizó un estudio encaminado a averiguarlo y a determinar si el porcentaje ha cambiado a lo largo del último decenio.

El estudio consistió en una revisión de los datos obtenidos mediante la Tercera y Cuarta Encuestas Nacionales de Evaluación de Salud y Nutrición (NHANES III y IV), que se realizaron entre 1988 y 1994 y entre 1999 y 2000, respectivamente. Estas encuestas tuvieron un diseño transversal y abarcaron una muestra nacional representativa de la población civil no recluida de los Estados Unidos. Los participantes fueron adultos mayores de 20 años con un diagnóstico de diabetes que hubieran sido entrevistados y sometidos a un examen físico ya fuese en NHANES III $(n=1265)$ o en NHANES IV $(n=441)$. Para expresar los resultados se utilizaron los valores de glucohemoglobina $\left(\mathrm{HbA}_{1 \mathrm{c}}\right)$, tensión arterial y colesterol total en suero, así como su relación con la presencia de trastornos vasculares.

En NHANES III y IV la proporción de pacientes con un diagnóstico previo de diabetes fue similar, independientemente de la edad o el sexo, pero los pacientes encuestados en NHANES IV mostraron una menor tendencia a ser blancos de origen no hispano, recibieron el diagnóstico de diabetes a una edad más temprana, tuvieron un mayor índice de masa corporal promedio y eran más proclives a emplear insulina en combinación con fármacos orales. Solo $37,0 \%$ de los participantes en NHANES IV lograron la meta de mantener las concentraciones de $\mathrm{HbA}_{1 \mathrm{c}}$ por debajo de $7,0 \%$, mientras que $37,2 \%$ de ellos tenían valores de $\mathrm{HbA}_{1 \mathrm{c}}$ superiores al recomendado como límite para "tomar acción" $(8,0 \%)$. No se encontraron diferencias significativas entre estos porcentajes y los detectados durante NHANES III ( $P=0,11$ y $P=0,67$, respectivamente). Solamente $35,8 \%$ de los participantes en NHANES IV mantenían la tensión arterial sistólica y diastólica por debajo de los valores recomendados $(130 \mathrm{~mm}$ $\mathrm{Hg}$ y de $80 \mathrm{~mm} \mathrm{Hg}$, respectivamente); 40,4\% tenían hipertensión arterial (tensión arterial sistólica $\geq 140$ $\mathrm{mmHg}$ o diastólica $\geq 90 \mathrm{~mm} \mathrm{Hg}$ ). Estos porcentajes no difierieron significativamente con respecto a los encontrados durante NHANES III $(P=0,10$ y $P=$ 0,56 , respectivamente). Más de la mitad $(51,8 \%)$ de los pacientes que participaron en NHANES IV presentaron concentraciones de colesterol total de 200 $\mathrm{mg} / \mathrm{dL}$ o más (frente a $66,1 \%$ en NHANES III; $P<$ 0,001 ). En general, solo 7,3\% (intervalo de confianza de $95 \%$ : 2,8 a 11,9\%) de los adultos con diabetes en NHANES IV alcanzaron la meta recomendada de mantener concentraciones de $\mathrm{HbA}_{1 \mathrm{c}}$ inferiores a $7 \%$, una tensión arterial por debajo de $130 / 80 \mathrm{~mm}$ de $\mathrm{Hg}$, y también concentraciones de colesterol menores de $200 \mathrm{mg} / \mathrm{dL}(5,18 \mathrm{mmol} / \mathrm{L})$.

Los resultados del estudio indican que hay que reforzar las medidas encaminadas a lograr que las personas con diabetes diagnosticada controlen mejor los factores de riesgo de sufrir trastornos vasculares.

Una limitación importante de este estudio fue que el reducido número de participantes con diagnóstico de diabetes en NHANES IV no permitió realizar el análisis por grupos de edad, raza, origen étnico o tipo de tratamiento. Además, el estudio solo abarcó a pacientes adultos no recluidos en instituciones. Por otra parte, las encuestas NHANES tienen un diseño transversal que permite sacar inferencias acerca de tendencias generales, pero no llegar a conclusiones sobre la relación temporal entre observaciones, puesto que los pacientes que participan en cada encuesta no son los mismos. (Saydah SH, Fradkin J, Cowie CC. Poor control of risk factors for vascular disease among adults with previously diagnosed diabetes. JAMA. 2004;291: 335-342.)

\section{Se estudia la relación entre la obesidad en la adolescencia y la mortalidad en la adultez}

Según se ha demostrado, la obesidad durante la niñez y la adolescencia es un importante factor que predispone a la obesidad durante la vida adulta y aumenta el riesgo de padecer diversas enfermedades y trastornos graves, entre ellos la diabetes y la enfermedad coronaria. Los efectos de la obesidad durante la adolescencia han sido poco estudiados, pero la prevalencia de diabetes tipo 2 entre niños y adolescentes de Estados Unidos de América parece guardar una relación directa con el índice de masa corporal (IMC). Se calcula que entre 50 y $80 \%$ de los adolescentes obesos se convierten en adultos obesos.

Se han realizado pocos estudios con seguimiento a largo plazo de la relación entre el IMC en la adolescencia y la adultez, o entre el IMC en la adolescencia y la mortalidad en la adultez. Esto se debe, entre otras cosas, a que la mortalidad en adultos jóvenes es baja, por lo que hacen falta estudios con un gran número de participantes para lograr resultados estadísticamente válidos.

En una investigación realizada en Noruega se estudió la relación entre el IMC durante la juventud 
y la adultez, así como la relación entre ambos indicadores por separado y el riesgo de morir. Para ello se tomaron el peso y la talla de 128121 adolescentes de 14 a 19 años de edad (61 522 hombres y 66599 mujeres) entre 1963 y 1999, y a cada persona se le hizo una segunda medición de manera estandarizada al cabo de 10 años o más. Se realizó un seguimiento promedio de 9,7 años (recorrido de 0 a 29 años) después de la segunda medición. Se emplearon modelos de riesgos instantáneos proporcionales de Cox para analizar la asociación entre el IMC obtenido durante la adolescencia y el obtenido durante la adultez, así como la asociación entre estos indicadores y la mortalidad.

La razón de posibilidades (OR) de ser obeso (IMC $\geq 30$ ) en la adultez aumentó progresivamente a medida que aumentó el IMC en la adolescencia. Las personas con valores de IMC muy altos durante la adolescencia mostraron un riesgo de morir al llegar a adultos de 30 a $40 \%$ mayor que las personas con un IMC moderado en la adolescencia. Sin embargo, al ajustar estos resultados según el IMC en la etapa adulta, no se encontró una asociación significativa entre la obesidad durante la adolescencia y el riesgo de morir en hombres adultos $(\mathrm{OR}=1,1$; intervalo de confianza de $95 \%$ [IC95\%]: 0,8 a 1,5), aunque sí en las mujeres (OR =1,3; IC95\%: 1,0 a 1,7). No se observaron diferencias cuando los resultados se ajustaron para controlar el efecto del hábito de fumar.

Como resultado de este trabajo se concluyó que la obesidad durante la adolescencia predispone a la obesidad durante la adultez. En adolescentes la obesidad se asocia con una mayor mortalidad posterior que parece obedecer fundamentalmente a las complicaciones de la obesidad en adultos. Los valores altos de IMC durante la adolescencia pueden constituir un elemento que predispone a la obesidad durante la vida adulta y a un mayor riesgo de morir prematuramente. (Engeland A, Bjorge T, Tverdal A, Sogaard AJ. Obesity in adolescence and adulthood and the risk of adult mortality. Epidemiology. 2004; 15(1):79-85).

\section{Se asocian la dureza del agua y la cantidad de fluoruro con una menor incidencia de infartos}

Numerosas investigaciones han establecido un vínculo entre la dureza del agua y la concentración de iones de fluoruro en ella con el riesgo de enfermedad coronaria. Sin embargo, hasta el momento no se ha demostrado que esa asociación sea estadísticamente significativa. En Finlandia, donde las aguas subterráneas tienden a ser suaves y los suelos y rocas se caracterizan por carecer de muchos oligoelementos esenciales, se ha observado que la distribución geográfica de un primer infarto agudo del miocardio (IAM) es heterogénea, y esta distribución espacial desigual es independiente de la incidencia de IAM.

Se realizó un estudio con el fin de evaluar la asociación entre la variación espacial de la incidencia de IAM y la composición geoquímica de las aguas subterráneas, en particular la dureza del agua $\left(\mathrm{en}^{\circ} \mathrm{dH}\right)$ y las concentraciones de calcio, magnesio, hierro e iones fluoruro y nitrato (en $\mathrm{mg} / \mathrm{L}$ ), así como de cobre, cinc y aluminio (en $\mu \mathrm{g} / \mathrm{L}$ ).

Para ello se realizó un estudio de pequeñas áreas mediante modelación bayesiana con datos geográficos agregados en cuadrículas de referencia de $10 \mathrm{~km} \times 10 \mathrm{~km}$. En total se estudío a 18946 hombres de 35 a 74 años de edad que habían sufrido un primer IAM, letal o no letal, en 1983, 1988 ó 1993. Los datos de la población se obtuvieron de Statistics Finland, la información acerca de los casos de IAM se obtuvo del Registro Nacional de Muertes y del Registro de Altas Hospitalarias, mientras que los datos geoquímicos se obtuvieron de la base hidrogeoquímica de la Encuesta Geológica de Finlandia.

Los resultados, ajustados por la edad, mostraron que la incidencia anual de IAM fue de 562,1 por 100000 hombres en las zonas donde la dureza del agua era menor de $1,7^{\circ} \mathrm{dH}$, frente a 469,5 por 100000 hombres en las áreas donde la dureza del agua estaba entre 1,71 y $5,2{ }^{\circ} \mathrm{dH}$, y a 437,6 por 100000 hombres en las áreas donde la dureza estaba por encima de $5,2{ }^{\circ} \mathrm{dH}$.

Cada aumento de $1^{\circ} \mathrm{dH}$ en la dureza del agua se asoció con una disminución de $1 \%$ del riesgo de IAM. Los elementos geoquímicos del agua subterránea no mostraron una asociación estadísticamente significativa con la incidencia de IAM o con la variación espacial de dicha incidencia, a pesar de los hallazgos que indican que el fluoruro tiene una acción protectora y que el hierro y el cobre favorecen la aparición de IAM.

Los resultados de este estudio, que utiliza un método de análisis más específico, confirman las conclusiones de investigaciones anteriores acerca de la relación inversa entre la dureza del agua y la enfermedad coronaria. Se debe estudiar más detalladamente el papel que desempeñan los factores geoquímicos ambientales en las variaciones geográficas de la incidencia de IAM. Para ello se recomienda incorporar los datos del consumo total de nutrientes, ya sea con los alimentos o con el agua. El análisis geoquímico espacial permite seleccionar las áreas apropiadas para este tipo de investigaciones. (Kousa A, Moltchanova E, Viik-Kajander M, Rytkonen $\mathrm{M}$, Tuomilehto J, Tarvainen T, et al. Geochemistry of ground water and the incidence of acute myocardial infarction in Finland. J Epidemiol Community Health. 2004 Feb; 58(2): 136-139.) 


\section{Las vacunas que contienen sales de aluminio son inocuas}

Durante décadas se han empleado compuestos de aluminio (sulfato potásico de aluminio, sulfato de aluminio e hidróxido de aluminio) en las vacunas para aumentar su inmunogenia y eficacia. Sin embargo, algunos autores han informado acerca de diversas reacciones adversas provocadas por vacunas que contienen aluminio, entre ellas reacciones en el sitio de la inyección con endurecimiento, abscesos estériles, nódulos subcutáneos, inflamación granulomatosa y eritema, así como un síndrome progresivo denominado miofascitis macrofágica caracterizado por desgaste muscular y agotamiento, lo que ha llevado a pensar en sustituir el aluminio en la composición de las vacunas. No obstante, los numerosos ensayos clínicos que harían falta para demostrar la inocuidad de las nuevas formulaciones de vacunas antes de poder emplearlas hacen que esta opción sea una empresa de gran envergadura y extremadamente costosa.

Debido a la dispersión y heterogeneidad de la información publicada sobre el tema, se decidió realizar una revisión sistemática con metaanálisis de los efectos adversos observados después de aplicar vacunas contra la difteria, el tétanos y la tos ferina (DTP) que contienen aluminio - ya sean monovalentes o combinadas- y compararlos con los observados después de emplear esas mismas vacunas sin aluminio o con menores concentraciones de este elemento. Para ello se realizaron búsquedas de estudios publicados sobre el tema en el registro del Cochrane Vaccine Field, la Cochrane Library, Medline, Embase, Biological Abstracts, Science Citation Index y el sitio en Internet del Sistema de Notificación de Reacciones Adversas Provocadas por Vacunas. La revisión abarcó todos los ensayos clínicos aleatorizados y semialeatorizados, así como estudios comparativos de cohorte, que ofrecieran suficiente información acerca de la concentración de aluminio, la composición de las vacunas y los resultados de los estudios de seguridad. Dos revisores extrajeron los datos de forma estandarizada de todos los estudios y evaluaron su calidad metodológica. De los 35 estudios identificados preliminarmente, 3 ensayos aleatorizados, 4 semialeatorizados y 1 estudio de cohorte cumplieron con los criterios de inclusión. El metaanálisis se realizó con los datos procedentes de cinco estudios relacionados con los dos aspectos principales que se deseaba comparar: las vacunas que contenían hidróxido de aluminio frente a las vacunas sin adyuvantes en niños de 18 meses de edad o menores, y vacunas que contenían otros compuestos de aluminio frente a vacunas sin adyuvantes en niños de 10 a 16 años.

En los niños más pequeños, las vacunas con hidróxido de aluminio causaron más eritema e in- duración que las vacunas sin adyuvante (razón de posibilidades $[\mathrm{OR}]=1,87$; intervalo de confianza de $95 \%$ [IC95\%]: 1,57 a 2,24) y menos reacciones de todo tipo (OR = 0,21; IC95\%: 0,15 a 0,28). En ambos casos la diferencia fue estadísticamente significativa. Las frecuencias de reacciones locales de todo tipo, así como de colapsos y convulsiones o de llantos persistentes o gritos, no difirieron de un modo significativo entre las dos cohortes de los ensayos. En niños mayores no se encontró ninguna asociación entre la exposición a vacunas que contenían aluminio y la aparición de induración, inflamación o hipertermia locales, aunque se observó una asociación con la presencia de dolor local de hasta 14 días de duración ( $\mathrm{OR}=2,05$; IC95\%: 1,25 a 3,38).

Aunque la inmunización con vacunas que contienen aluminio estuvo asociada con un mayor riesgo de enrojecimiento en el sitio de la inyección en los niños menores y con dolor local prolongado en niños mayores, no se encontraron pruebas de que las vacunas con sales de aluminio provocaran trastornos graves o de larga duración.

A pesar de la baja calidad de los estudios analizados, no se encontraron pruebas que respalden la necesidad de emprender nuevas investigaciones sobre este tema ni que apoyen una eventual decisión de sustituir las sales de aluminio en la composición de las vacunas. (Jefferson T, Rudin M, Di Pietrantonj $\mathrm{C}$. Adverse events after immunisation with aluminium-containing DTP vaccines: systematic review of the evidence. Lancet Infec Dis. 2004;4(2). Puede consultarse en: http://infection.thelancet. com/journal/vol4/iss2/full/laid.4.2.review_and_ opinion.28537.1. Acceso el 7 de febrero de 2004.)

\section{Una prueba sencilla determina el riesgo cardíaco en diabéticos}

Diversas investigaciones han demostrado que la capacidad de dilatación coronaria que depende del endotelio vascular permite predecir de manera independiente el riesgo de incidentes cardiovasculares en pacientes con varios factores de riesgo de enfermedad coronaria.

Para evaluar la capacidad de dilatación de los vasos coronarios del epicardio se ha utilizado la prueba de crioestimulación vasotensora, que consiste en sumergir las manos del paciente en agua helada durante dos minutos. Este procedimiento debe provocar que las arterias coronarias se dilaten. Sin embargo, se ha observado que en pacientes diabéticos la reacción es la inversa y las arterias coronarias se contraen.

Recentiemente se efectuó una investigación para determinar el efecto en pacientes con diabetes tipo 2 de la prueba de crioestimulación vasotensora. 
Se examinaron 128 personas: 72 pacientes con diabetes tipo 2 (edad promedio de 50,3 \pm 8,5 años) sin otro factor de riesgo coronario de importancia y 56 personas sin afecciones conocidas que sirvieron de testigos (edad promedio de 51,7 $\pm 6,4$ años). La respuesta de los participantes a la prueba de crioestimulación vasotensora se evaluó mediante angiografía coronaria cuantitativa. Se realizó un seguimiento de $45 \pm 19$ meses para determinar la frecuencia de incidentes cardiovasculares durante ese período.

El cambio promedio del diámetro de las arterias coronarias en el grupo testigo fue de 17,2 \pm $10,4 \%$; en $9,1 \%$ de los testigos hubo dilatación y en $8,9 \%$ no se constataron cambios, pero en ningún caso se observó la constricción de las coronarias. En los pacientes diabéticos, el cambio promedio del diámetro coronario fue de $-4,4 \pm 12,1 \%$, cifra significativamente menor que la del grupo testigo $(P<$ 0,001 ); en $73,6 \%$ de los pacientes se observó la constricción de las arterias coronarias, en $26,4 \%$ no se observaron cambios y en ningún caso se detectó la dilatación de las coronarias.

Durante el seguimiento ocurrió un solo incidente cardiovascular en el grupo testigo, en compa- ración con 26 en 18 de los 72 pacientes diabéticos $(P<0,001)$. Se constataron 23 incidentes cardiovasculares en 16 de los 53 pacientes diabéticos que reaccionaron a la prueba de crioestimulación vasotensora con la constricción de las coronarias, y la diferencia entre este grupo y el grupo testigo fue significativa $(P<0,001)$. Se observaron, además, tres incidentes cardiovasculares en 2 de los 19 pacientes diabéticos en quienes no se observaron cambios en el diámetro de las arterias coronarias durante la prueba (diferencia no significativa en relación con el grupo testigo).

Los resultados obtenidos demuestran que la constricción de las arterias coronarias, evaluada mediante estudios angiográficos, como respuesta a la prueba de crioestimulación vasotensora tiene valor pronóstico a largo plazo con respecto a los incidentes cardiovasculares en pacientes con diabetes tipo 2 sin otros factores de riesgo coronario de importancia. (Nitenberg A, Valensi P, Sachs R, Cosson E, Attali JR, Antony I. Prognostic value of epicardial coronary artery constriction to the cold pressor test in type 2 diabetic patients with angiographically normal coronary arteries and no other major coronary risk factors. Diabetes Care 2004;27:208-215.)
DESAFIOS

PARA LA

EDUCACIÓN EI

SALUD

PÚBLICA

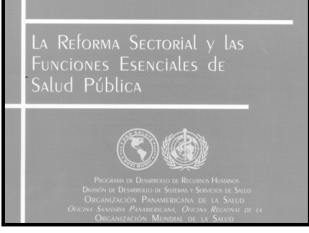

1999, 471pp.

ISBN 9283204077

Código: OT 113

Precios: US\$ 24,00/

US\$ 18,00 en América Latina y el Caribe

\section{Desafíos para la educación en salud pública. La reforma sectorial y las funciones esenciales de salud pública}

Esta publicación es expresión de la riqueza del debate que se dió en la II Conferencia Panamericana de Educación Pública y recoge los textos de las conferencias magistrales de los distinguidos exponentes y los comentarios a las mismas.

Esta conferencia convocó a relevantes actores tanto educacionales como de servicio en el campo de la salud pública, con el propósito de discutir y diseñar las estrategias de desarrollo de los recursos humanos en salud pública apropiadas a las exigencias de las Funciones Esenciales de Salud Pública (FESP) y los procesos de reforma sectorial, incluido el desarrollo de la Rectoría de los Ministerios de Salud, fundamentalmente para construir una nueva agenda de la educación en salud pública y de la cooperación técnica en este campo.

Usted también puede enviar su pedido y pago en dólares estadounidenses a: http://publications.paho.org Fax: (301) 206-9789・E-mail: paho@pmds.com・Oficina de la OPS/OMS en su país 